www.nature.com/bmt

\title{
Editorial
}

\section{High-dose therapy in breast cancer: out of favor but not out of promise}

\author{
S Glück and D Stewart \\ Tom Baker Cancer Centre, University of Calgary, Calgary, Canada
}

\section{Summary:}

The evidence for use of high-dose chemotherapy and autologous stem cell transplantation for breast cancer still remains inconclusive at best. A number of prospective randomized phase III studies have been either published or presented recently or are underway in North America and Europe. It will be crucial to complete the available prospective randomized phase III study and obtain the data when all studies reach a mature status. Only then will level I evidence become available to determine the efficacy and effectiveness of high-dose chemotherapy and autologous stem cell transplantation in breast cancer. Bone Marrow Transplantation (2000) 25, 1017-1019.

Keywords: high-dose chemotherapy; breast cancer; evidence for efficacy; controversy

The use of high-dose chemotherapy (HDCT) and autologous stem cell transplantation (ASCT) for breast cancer has steadily increased over the last decade. The Autologous Blood and Marrow Transplantation Registry (ABMTR) reported over 3000 cases in 1996. ${ }^{1}$ This increase is due to encouraging results from a variety of phase I and phase II studies, one randomized prospective phase III study published in $1995^{2}$ and a number of retrospective and contemporary analyses. ${ }^{3}$ At the same time, the mortality due to the treatment steadily decreased from over $15 \%$ to $2-3 \%{ }^{2}$ The toxicity, too, decreased mainly due to the use of peripheral blood (rather than bone marrow) as a source of stem cells, the use of modern supportive care including hematopoietic growth factors and better patient selection. A number of phase III studies were initiated in the early and mid-1990s, but accrual was slow due to the fact that in the United States this promising treatment was offered to patients outside clinical trials long before enough evidence became available to support such a decision. European and Canadian institutions demanded more evidence to justify such

Correspondence: Dr S Glück, Dept Oncology, Medicine and Pharmacology and Therapeutics, Faculty of Medicine, University of Calgary, Medical Oncologist, Tom Baker Cancer Centre, 1331-29 Street NW, Calgary, Alberta T2N 4N2, Canada

Received 1 October 1999; accepted 31 January 2000 toxic and potentially dangerous treatment. Results from a number of phase III trials evaluating HDCT and ASCT for breast cancer have recently become available. These results are summarized below.

\section{Metastatic breast cancer}

A study presented by Lotz et $a l,{ }^{4}$ shows a median time to progression of 15.7 vs 26.9 months $(P=0.04)$. After 2 years, a lower proportion of patients treated with high-dose chemotherapy had relapsed than had those treated with conventional chemotherapy (27\% vs 52\%). Overall survival was not significantly different between the groups. However a trend for longer median overall survival with the intensive regimen (16 vs 36 months) was observed. These differences are clinically relevant but statistically not significant due to the fact that the sample size was too low (only 61 patients accrued into this study, PEGASE 04) to have the power to detect any statistically meaningful difference in survival.

Stadtmauer et $a l^{5}$ presented a study of 199 patients with metastatic breast cancer that has sufficient statistical power to detect a difference in survival of $20 \%$ at 2 years and a median follow-up of 37 months. This trial did not show any benefit of alkylator-based HDCT over SDCT in terms of event-free survival (6 vs 10 months) or overall survival (24 vs 26 months).

The study published by Bezwoda et $a l^{2}$ in 1995 clearly demonstrates the advantage of mitoxantrone-based HDCT and ASCT over the standard treatment, as used by the South African authors. Both progression-free survival and overall survival are statistically better with clinically meaningful advantage. Obviously, this study has a few flaws, for example unusual standard treatment that is not being used in North America or Europe; a rather small sample size of 45 in each arm, and tamoxifen intervention which was more common in the HDCT arm.

Lastly, Peters et $a l^{6}$ presented a randomized prospective study of alkylator-based HDCT for patients with metastatic breast cancer at the 1996 American Society of Clinical Oncology meeting. Although the study found a statistically significant difference in outcome between treatment arms, it did not compare standard treatment vs HDCT but instead compared immediate $v s$ delayed HDCT and ASCT after reaching complete remission to AFM non-alkylator induc- 
tion chemotherapy. Interestingly, delayed HDCT (providing a treatment-free interval) resulted in superior survival to high-dose consolidation immediately after induction chemotherapy.

One of the above-mentioned studies is 'positive'; one is negative showing only minimal differences in outcomes; one shows a positive trend (with statistically significant difference for time to progression), but does not have enough power to detect any significant differences and one study does not compare HDCT vs SDCT. In spite of these results, the question regarding the use of HDCT and ASCT in patients with metastatic breast cancer remains unanswered and therefore controversial. The National Cancer Institute of Canada Clinical Trials Group designed a study, NCI-C CTG MA $16,{ }^{7}$ to answer the question of whether or not consolidation treatment with HDCT and ASCT can improve survival relative to what is presently considered standard treatment for first-line therapy of metastatic breast cancer in North America and Europe. The study was initially designed to have $80 \%$ power to detect a difference of $20 \%$ of overall survival at 2 years. By June 1999, approximately 1 year ahead of time, the fully calculated sample size was reached. During the NCIC CTG Spring meeting in Toronto, the Breast Cancer Committee agreed to extend the study by approximately 12 months, anticipating an additional 100 patients to be enrolled on to the study. A smaller, $13 \%$ difference in survival at 2 years could then be detected with the power of $80 \%$ with such sample size increment. Currently, no other active study in North America has the potential to scientifically solve the question of consolidation treatment with HDCT and ASCT in patients with metastatic breast cancer. The extended NCIC-CTG MA16 does have the potential to answer this important clinical question.

\section{HDCT and ASCT as adjuvant treatment}

Following results from four different randomized prospective phase III studies in patients with high risk stage II breast cancer (usually 10 or more axillary lymph nodes involved with metastatic disease) have been presented or published.

Peters et $a l^{8}$ presented the data on 783 randomized patients, treated with four cycles of CAF followed by one cycle of $\mathrm{cDDP} / \mathrm{CTX} / \mathrm{BCNU}$ either using high dosing and autologous stem cell rescue or using doses that require only G-CSF support. At 3 years, the differences in event-free survival and overall survival are not statistically different (68\% vs 64\% and 78 vs $80 \%$ respectively). In this patient population, clinical studies require a much longer followup. As an example, results of studies that eventually proved the effectiveness of HDCT and ASCT in multiple myeloma or non-Hodgkin's lymphoma, initially were negative at 2 3 year follow-up. In the adjuvant setting, many studies in patients with breast cancer have shown positive results only after 5 or more years observation time. Peters' study ${ }^{8}$ is too early to deliver final results, does not incorporate particularly active breast cancer drugs in the HDCT regimen, and has a very high incidence of treatment-related deaths in the high-dose arm of the study (7.5\%). Another observation needs to be addressed: the 'standard' arm of the study shows a much better outcome than similar ones would predict. The likely explanation is 'stage migration' and affects the ability to detect improvement in outcome, particularly with short follow-up. ${ }^{9}$

The Scandinavian breast cancer study group, ${ }^{10}$ reported their trial of 525 patients in the same patient population as Peters et al. They compared directly three cycles of FEC followed by one cycle of high-dose $\mathrm{CTCb}$ and ASCT to the so-called 'standard arm', consisting of nine cycles of 'tailored' FEC supported by G-CSF. At 20 months, they reported 78 relapses in the 'tailored FEC' and 50 relapses in the HDCT arm. These differences are not significant. At the same time, 40 deaths have been observed in each arm. In the non-transplant arm, a few patients developed secondary acute leukemia and myelodysplasias. This very balanced study is obviously comparing two different doseintensified regimens, rather than one HDCT and ASCT to conventional/standard treatments. The observation of secondary hematological malignancies is worrying. Follow-up is again too short to draw final conclusions.

Rodenhuis et $a l^{11}$ published a study in 1998 in the Lancet, comparing four cycles of FEC at a standard dose to one cycle FEC plus one cycle of CTCb followed by ASCT. The patients all had the apex of the axillary lymph nodes positive for involvement with breast cancer. Only 81 patients were randomized and are evaluable: EFS is $46 \mathrm{vs}$ $47 \%$ and overall survival 72 vs $76 \%$. This study certainly has not enough power to detect any meaningful clinical or statistical differences. The patient population also is somewhat different from those in the other studies.

Hortobagyi et al ${ }^{12}$ presented a study in 1998. Seventyeight patients with high-risk breast cancer (as defined by either $\geqslant 10$ lymph nodes positive after resectable breast cancer or $\geqslant 4$ lymph nodes positive after neo-adjuvant chemotherapy). All patients received eight cycles of FAC, $50 \%$ were randomized to receive two cycles of HDCT (using VP16, cyclophosphamide and cisplatinum) followed by ASCT. The 4-year disease-free survival was $52 \%$ and $51 \%$ (by actual treatment received, $P=0.84$ ) and overall survival was $64 \%$ vs $63 \%$ (by actual treatment received, $P$ $=0.66$ ) which is not statistically different in both groups. The authors conclude that because of the modest sample size this trial has a limited statistical power. However, the data suggest that HDCT with ASCT as consolidation after eight cycles of adjuvant $\mathrm{CT}$ is unlikely to produce major improvements over FAC alone.

Bezwoda et $a l^{13}$ presented a study randomising 154 patients with stage II breast cancer involving 10 or more lymph nodes. Patients who received chemotherapy with ASCT fared significantly better than those in the control arm. However according to the article by Richard Horton in The Lancet 2000; 355: 942-943, this study was re-evaluated and the original data could not be confirmed. The study may have indeed been carried out in a fraudulent manner. Thus, this should be considered when referring to the data and conclusions of the paper.

In the adjuvant setting, more and better designed studies are necessary to clearly show whether HDCT and ASCT is superior treatment to 'standard' chemotherapy in patients with high risk stage II breast cancer. The Bezwoda trial 
result demands immediate confirmation by a major cooperative group.

\section{Conclusions}

To date, no definite answers are available to the initial question, whether or not HDCT and ASCT can improve quantity or quality of life. Completing the available prospective, randomized phase III studies, using HDCT earlier in the course of treatment, applying drugs that are active in breast cancer at standard doses, comparing the experimental treatment to standard therapy and using appropriate sample size to detect clinically meaningful and statistically significant differences are all paramount requirements to answer the question successfully. Within the next few years, a number of European ${ }^{14}$ and two Canadian Studies ${ }^{7,15}$ that are currently accruing patients, will contribute important information regarding the role of HDCT and ASCT for breast cancer.

\section{References}

1 Autologous Blood and Marrow Transplant Registry. Medical College of Wisconsin, Milwaukee, WI, 53226 USA, 1996.

2 Bezwoda WR, Seymore L, Dansey RD. High-dose chemotherapy with hematopoietic rescue as primary treatment or metastatic breast cancer: a randomized trial. J Clin Oncol 1995; 13: 2483-2489.

3 Glück S, Crump M, Bociek G, Stewart D. High dose chemotherapy with autologous blood stem cell transplantation: increasing evidence for efficacy in patients with metastatic breast cancer. Autologous Blood and Marrow Transplantation, Proceedings of the 9th International Symposium, Arlington, Texas, 1999, pp 290-302.

4 Lotz JP, Cure H, Janvier M et al. High dose chemotherapy with hematopoietic stem cells transplantation for metastatic breast cancer: results of the French protocol PEGASE 04. Proc Am Soc Clin Oncol 1999; 18: 161a.

5 Stadtmauer EA, O'Neill A, Goldstein LJ et al. Phase III randomized trial of high dose chemotherapy and stem cell support shows no difference in overall survival or severe toxicity compared to maintenance chemotherapy with cyclophosphamide, methotrexate and 5-fluorouracil for women with metastatic breast cancer who are responding to conventional induction chemotherapy: the Philadelphia intergroup study (PBT-1).

6 Peters WP, Jones RB, Vredenburgh J et al. A large, prospective, randomized trial of high-dose combination alkylating agents (CPB) with autologous cellular support (ABMS) as consolidation for patients with metastatic breast cancer achieving complete remission after intensive doxorubicin-based induction therapy (AFM). Proc Am Soc Clin Oncol 1996; 15: 149 .

7 Glück S, Crump M. A randomised trial of high-dose chemotherapy and autologous stem cell therapy $v s$ standard therapy in women with metastatic breast cancer who have responded to anthracycline or taxane-based induction chemotherapy, National Cancer Institute of Canada Clinical Trials Group. NCIC CTG Protocol Number MA. 16, 1997.

8 Peters WP, Rossner G, Vredenburgh J et al. A prospective randomized comparison of two doses of combination alkylating agents as consolidation after $\mathrm{CAF}$ in high risk primary breast cancer involving ten or more axillary lymph nodes: preliminary results of CALGB 9082 hSWOG9114 hNCICMA113. Proc Am Soc Clin Oncol 1999; 18: 2 a.

9 Crump M, Goss PE, Prince M, Girouard C. Outcome of extensive evaluation prior to adjuvant therapy in women with breast cancer and $\geqslant 10$ positive axillary nodes. J Clin Oncol 1996; 14: 66-69.

10 The Scandinavian Breast Cancer Study Group 9401. Results from a randomized adjuvant breast cancer study with highdose chemotherapy with $\mathrm{CTCb}$ supported by autologous bone marrow stem cells $v s$ dose escalated and tailored FEC therapy. Proc Am Soc Clin Oncol 1999; 18: 3a.

11 Rodenhuis S, Richel DJ, van der Wall et al. Randomized trial of high dose chemotherapy and haematopoietic progenitor-cell support in operable breast cancer with extensive axillary lymph node involvement. Lancet 1998; 325: 515-521.

12 Hortobagyi GN, Buzdar AU, Champlin R, Gajewski J. Lack of efficacy of adjuvant high dose (HD) tandem combination chemotherapy (CT) for high risk primary breast cancer - a randomised trial. Proc Am Soc Clin Oncol 1998; 17: 471.

13 Bezwoda WR. Randomized controlled trial of high dose chemotherapy versus standard dose chemotherapy for high risk, surgically treated primary breast cancer. Proc Am Soc Clin Oncol 1999; 18: 4a.

14 Rosti G. European Bone Marrow Transplantation Group. Chair: solid tumors. Ravenna, Italy.

15 A multicentre phase III randomised trial comparing docetaxel in combination with doxorubicin and cyclophosphamide (TAC) versus TAC followed by high dose chemotherapy with mitoxantrone, cyclophosphamide and vinorelbine with autologous peripheral stem cell transplantation and G-CSF in adjuvant treatment of operable breast cancer with four or more positive axillary nodes. TAX 321 (BCIRG 002). 